\title{
Macroscopic quantum self-trapping and Josephson oscillations of exciton polaritons
}

\author{
M. Abbarchi ${ }^{1,2}$, A. Amo ${ }^{1 \star}$, V. G. Sala ${ }^{1,3}$, D. D. Solnyshkov ${ }^{4}$, H. Flayac ${ }^{4}$, L. Ferrier ${ }^{1}$, I. Sagnes ${ }^{1}$, \\ E. Galopin', A. Lemaître ${ }^{1}$, G. Malpuech ${ }^{4}$ and J. Bloch ${ }^{1}$
}

The coupling of two macroscopic quantum states through a tunnel barrier gives rise to Josephson phenomena ${ }^{1}$ such as Rabi oscillations ${ }^{2}$, the a.c. and d.c. effects ${ }^{3}$, or macroscopic self-trapping, depending on whether tunnelling or interactions dominate ${ }^{4}$. Nonlinear Josephson physics was first observed in superfluid helium ${ }^{5}$ and atomic condensates ${ }^{6,7}$, but it has remained inaccessible in photonic systems because it requires large photon-photon interactions. Here we report on the observation of nonlinear Josephson oscillations of two coupled polariton condensates confined in a photonic molecule formed by two overlapping micropillars etched in a semiconductor microcavity ${ }^{8}$. At low densities we observe coherent oscillations of particles tunnelling between the two sites. At high densities, interactions quench the transfer of particles, inducing the macroscopic self-trapping of polaritons in one of the micropillars ${ }^{9,10}$. The finite lifetime results in a dynamical transition from self-trapping to oscillations with $\pi$ phase. Our results open the way to the experimental study of highly nonlinear regimes in photonic systems, such as chaos ${ }^{11-13}$ or symmetry-breaking bifurcations ${ }^{14,15}$.

A bosonic Josephson junction is a device in which two macroscopic ensembles of bosons, each of them occupying a single quantum state, are coupled by a tunnel barrier. The system can be described by the following coupled nonlinear Schrödinger equations ${ }^{1}$ :

$$
\begin{aligned}
& i \hbar \frac{\mathrm{d} \psi_{\mathrm{L}}}{\mathrm{d} t}=\left(E_{\mathrm{L}}^{0}+U\left|\psi_{\mathrm{L}}\right|^{2}\right) \psi_{\mathrm{L}}-J \psi_{\mathrm{R}} \\
& i \hbar \frac{\mathrm{d} \psi_{\mathrm{R}}}{\mathrm{d} t}=\left(E_{\mathrm{R}}^{0}+U\left|\psi_{\mathrm{R}}\right|^{2}\right) \psi_{\mathrm{R}}-J \psi_{\mathrm{L}}
\end{aligned}
$$

where $\psi_{\mathrm{L}, \mathrm{R}}$ are the bosonic wavefunctions with particle densities $\left|\psi_{\mathrm{L}, \mathrm{R}}\right|^{2}$ localized to the left (L) and to the right ( $\left.\mathrm{R}\right)$ of the barrier, $E_{\mathrm{L}, \mathrm{R}}^{0}$ is the single particle energy of the quantum states, $U$ is the particle-particle interaction strength and $J$ is the tunnel coupling constant. In the absence of interactions, equations (1a) and (1b) can be diagonalized in a basis of bonding $\left(2^{-1 / 2}\left[\psi_{\mathrm{L}}+\psi_{\mathrm{R}}\right]\right)$ and antibonding states $\left(2^{-1 / 2}\left[\psi_{\mathrm{L}}-\psi_{\mathrm{R}}\right]\right)$. An initial state prepared in a linear combination of these two (for instance, all particles in the left site) will result in density oscillations between the two sites. This is the main principle of the bosonic Josephson effect, which manifests in an ensemble of oscillatory regimes. In the absence of interactions, sinusoidal oscillations take place ${ }^{4,7}$ with a frequency $\hbar \omega=\sqrt{4 J^{2}+\left(E_{\mathrm{L}}^{0}-E_{\mathrm{R}}^{0}\right)^{2}}$.
Josephson physics shows the most spectacular phenomena in the nonlinear regime, when the interaction energy $\left(U|\psi|^{2}\right)$ is greater than the coupling $J$. The transfer of particles from one site to the other gives rise to a dynamical renormalization of the energy in each site, resulting in anharmonic oscillations. If interactions are strong enough $\left(U|\psi|^{2} \gg J\right)$, the self-induced energy renormalization quenches the tunnelling, and most of the particles remain localized in one of the sites. This out of equilibrium metastable regime is called macroscopic quantum self-trapping.

A number of bosonic systems have demonstrated Josephson physics. Harmonic oscillations in the linear regime have been observed in superconductor junctions ${ }^{2}$ or in nanoscale apertures connecting superfluid helium vessels ${ }^{5}$. Bose-Einstein condensates of ultracold atoms in coupled traps have, in addition, shown macroscopic self-trapping 6 , and collective phases in arrays of junctions ${ }^{16}$. For photonic systems, mostly theoretical results have been obtained so far. Despite the observation of symmetry breaking in photonic Kerr media ${ }^{17,18}$, nonlinear Josephson oscillations ${ }^{9,10,19}$ have remained inaccessible owing to the short lifetimes and very weak effective photon-photon interactions in standard nonlinear optical systems.

In this work we present the experimental observation of nonlinear Josephson physics in a highly nonlinear photonic system, namely cavity polaritons confined in coupled micropillars ${ }^{8}$. Polaritons are quasiparticles arising from the strong coupling between quantum well excitons and photons confined in a semiconductor microcavity. Their mixed light-matter nature allows one, on one hand, to create a well-controlled double well by engineering their photonic component and, on the other hand, to boost the optical nonlinearities thanks to the strongly interacting excitonic component. In our structures, the two micropillars in which polaritons are confined, partially overlap (Fig. 1), creating a bosonic Josephson junction. Polaritons can tunnel from one site to the other with a coupling strength $J$, determined by the centre-to-centre separation ${ }^{8}$. Coherent oscillations in the linear regime between two coupled polariton condensates have been recently observed in a planar CdTe microcavity using two neighbouring photonic defects present in the random disorder of the structure ${ }^{20}$. Here we show the passage from coherent oscillations between the two sites with controlled frequency, to the extreme nonlinear situation of trapping in a single micropillar induced by self-interactions. As polaritons have a finite lifetime, the interaction energy changes dynamically and we observe anharmonic phases, and the transition in time from the self-trapped to the oscillatory regime.

\footnotetext{
${ }^{1}$ Laboratoire de Photonique et Nanostructures, LPN/CNRS, Route de Nozay, 91460 Marcoussis, France, ${ }^{2}$ Laboratoire Pierre Aigrain, École Normale Supérieure, CNRS (UMR 8551), Université Pierre et Marie Curie, Université D. Diderot, 75231 Paris Cedex 05, France, ${ }^{3}$ Laboratoire Kastler Brossel, Université Pierre et Marie Curie, École Normale Supérieure et CNRS, UPMC Case 74, 4 place Jussieu, 75252 Paris Cedex 05, France, ${ }^{4}$ Institut Pascal, PHOTON-N2, Clermont Université, University Blaise Pascal, CNRS, 24 avenue des Landais, 63177 Aubière Cedex, France. *e-mail: alberto.amo@lpn.cnrs.fr.
} 
a

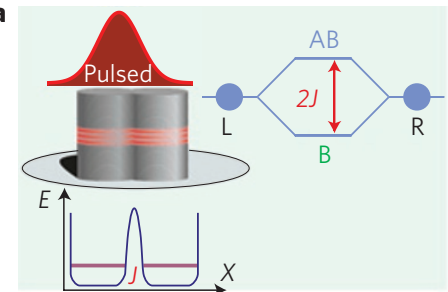

d

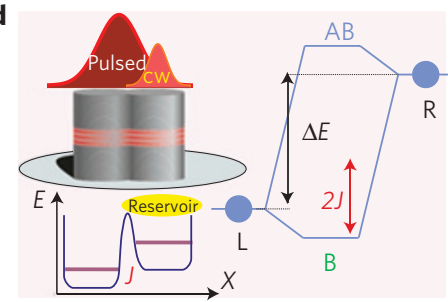

b

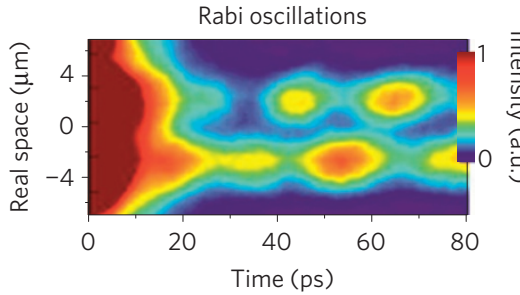

e

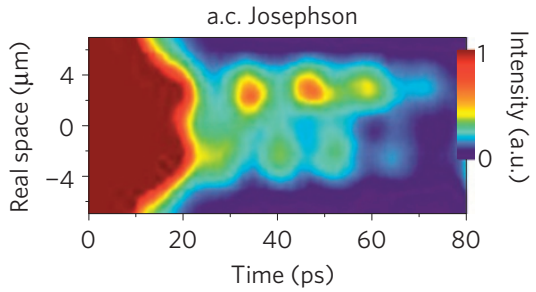

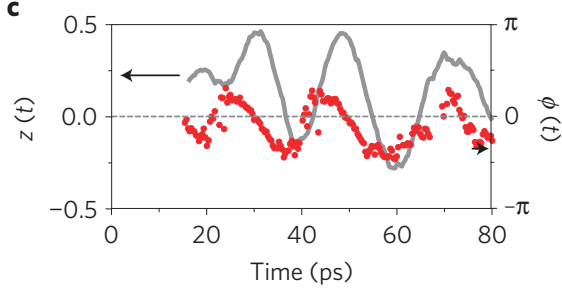

f

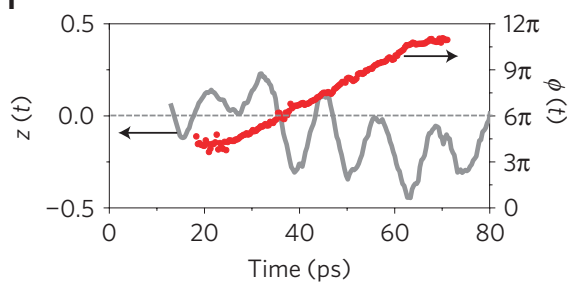

Figure 1 | Rabi oscillations and a.c. Josephson effect. a, A polaritonic molecule. The coupling $J(0.1$ meV) between the lowest energy state (ground state) of each micropillar ( $L, R)$ gives rise to bonding $(B)$ and antibonding ( $A B$ ) modes. b. Emitted intensity when an off-centred Gaussian pulse at low power $(2.5 \mathrm{~mW})$ excites the system. c, Measured population imbalance (grey line) and phase difference (red dots), showing harmonic oscillations with a frequency given by $\hbar \omega=2 \mathrm{~J}$. The slight asymmetry of the oscillations about $z=0$ might be caused by an unintentional difference in the size of the micropillars. d, The a.c. Josephson regime is achieved by adding a cw beam on top of the right micropillar, which creates a reservoir (shown in yellow) inducing a static blueshift of its ground state energy. e-f, The larger bonding-antibonding splitting results in faster intensity oscillations (e), and in a monotonously increasing phase difference (f, red points).

The coupled micropillars used in our studies (Fig. 1a) are obtained by dry etching of a planar semiconductor microcavity with a Rabi splitting of $15 \mathrm{meV}$ at $10 \mathrm{~K}$, the temperature of our experiments (see Methods). Each individual pillar has a diameter of $4 \mu \mathrm{m}$ and presents a series of confined polaritonic states ${ }^{21-23}$ with a lifetime $\tau \sim 33$ ps. The centre-to-centre separation of $3.7 \mu \mathrm{m}$ results in a tunnel coupling of the lowest energy confined polaritonic states (ground state) of $J=0.1 \mathrm{meV}$. This double potential well system can be described by equations (1a) and (1b) with the addition of a phenomenological decay term ${ }^{9,24},-i(\hbar / 2 \tau) \psi_{\mathrm{L}(\mathrm{R})}$, accounting for the polariton losses due to the escape of photons out of the cavity, and with a positive value of $U$ coming from the polariton-polariton repulsive interactions ${ }^{25}$.

The Madelung transformation $\psi_{\mathrm{L}, \mathrm{R}}(t)=\sqrt{N_{\mathrm{L}, \mathrm{R}}(t)} \mathrm{e}^{i \theta_{\mathrm{L}, \mathrm{R}}(t)}$ allows us to rewrite equations (1) in their dynamical form ${ }^{4}$ :

$$
\begin{gathered}
\frac{\hbar}{2 J} \dot{z}=\sqrt{1-z^{2}(t)} \sin \phi(t) \\
-\frac{\hbar}{2 J} \dot{\phi}=\frac{E_{\mathrm{L}}^{0}-E_{\mathrm{R}}^{0}}{2 J}+\frac{U N_{\mathrm{T}} \mathrm{e}^{-t / \tau}}{2 J} z(t)+\frac{z(t)}{\sqrt{1-z^{2}(t)}} \cos \phi(t)
\end{gathered}
$$

where $z(t)=\frac{N_{\mathrm{L}}-N_{\mathrm{R}}}{N_{\mathrm{T}}}$ is the population imbalance between the two micropillars (with $N_{\mathrm{T}}=N_{\mathrm{L}}+N_{\mathrm{R}}$ the total population), and $\phi(t)=\theta_{\mathrm{L}}(t)-\theta_{\mathrm{R}}(t)$ is the phase difference. $E_{\mathrm{L}}^{0}-E_{\mathrm{R}}^{0}$ is the energy difference between the ground states of the left and right micropillars in the absence of coupling, negligible in our case as the two pillars are nominally identical. The second term on the right hand side of equation (2b) contains all the features due to interactions, and it is the only one that is affected by the polariton finite lifetime.

To study the different Josephson regimes we excite the system with a 1.7 ps pulsed laser resonant with the ground state energy of the micropillars $(\sim 780 \mathrm{~nm})$. The spectral width of the laser $(0.4 \mathrm{meV}>J)$ allows us to initialize the system in a linear combination of bonding and antibonding $\operatorname{states}^{26}(\mathrm{~B}$ and $\mathrm{AB}$ in the figures). We use a $10 \mu \mathrm{m}$ wide Gaussian excitation spot, covering the whole molecule. In this geometry, $\phi(0)=0$ and the initial population imbalance $z(0)$ can be tuned by shifting the spot with respect to the centre of the molecule.
The light emitted from a single polaritonic molecule is collected with a microscope objective in reflection geometry and analysed in energy and time by means of a spectrometer coupled to a streak camera. To avoid the strong reflection of the laser beam in our detectors, we select the linear polarization of emission that is perpendicular to that of the excitation (parallel to the molecule long axis). The molecules present an intrinsic linear polarization splitting along a non-trivial direction which slowly rotates the polarization direction of the injected polaritons ${ }^{27}$. This allows us to measure in cross-polarized detection the energy, population imbalance $z(t)$ and phase difference $\phi(t)$ between the two sites (see Methods). The polarization splitting is of the order of $\sim 40 \mu \mathrm{eV}$, resulting in a polarization rotation that is much slower $(>70 \mathrm{ps})$ than the Josephson oscillation timescales in our system (up to $21 \mathrm{ps}$ ).

At low excitation density $\left(2 J \gg U N_{\mathrm{T}} \approx 0\right)$, interaction effects are negligible and the dynamics of the system is entirely dominated by the tunnel coupling. This is the situation presented in Fig. 1a-c: coherent oscillations of the population and phase are observed when the initial population imbalance is $z(0)=0.45$. The measured oscillation period of $21 \mathrm{ps}$ coincides with that expected for the nominal coupling of $J=0.1 \mathrm{meV}$ in this molecule. The periodic oscillations of both population and phase around zero correspond to the regime of Rabi oscillations of two coupled modes with $\Delta E \equiv E_{\mathrm{L}}^{0}-E_{\mathrm{R}}^{0} \approx 0$.

A different regime, characterized by a running phase, can be accessed by inducing an energy splitting $E_{\mathrm{L}}^{0}-E_{\mathrm{R}}^{0} \gtrsim J$ between the ground states of the micropillars. To do so, we add a weak continuous wave $(\mathrm{cw})$ non-resonant beam $(730 \mathrm{~nm})$ focussed onto one of the micropillars (the right one, see Fig. 1d-f). This beam creates an excitonic reservoir which interacts with the ground state polariton mode of that micropillar, inducing a stationary and local energy blueshift of about $0.35 \mathrm{meV}$ (see Supplementary Fig. S2). Nevertheless, particle self-interactions (within the condensates) remain negligible $\left(U N_{\mathrm{T}} \approx 0\right)$. Figure $1 \mathrm{e}$, f shows that the addition of this beam results in an acceleration of the oscillations, and in a phase difference $\phi(t)$ which monotonously increases with time, well reproduced by our simulations (see section D of the Supplementary Information). This regime is analogous to the socalled a.c. Josephson effect, in which a constant voltage difference 
a

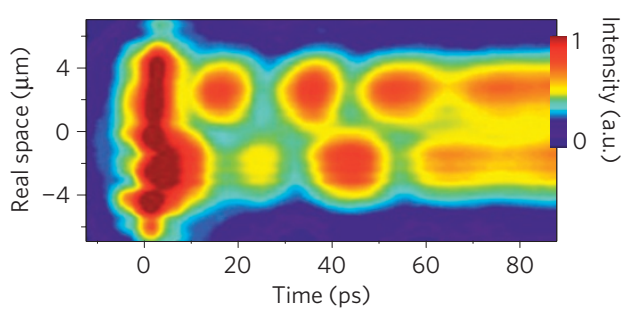

b
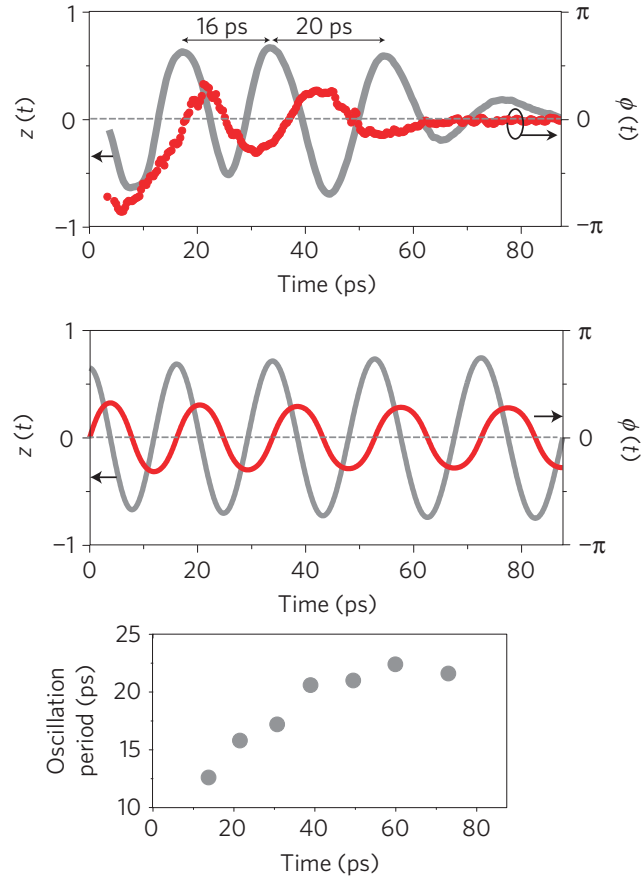

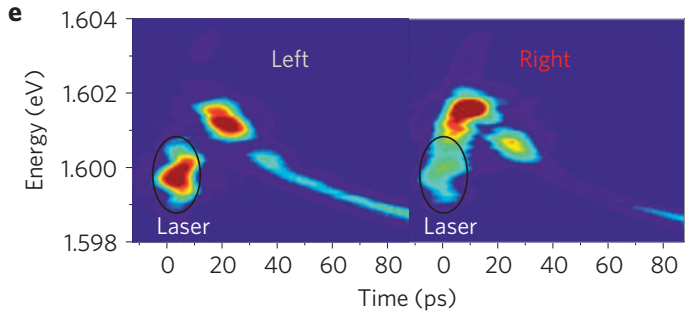

f

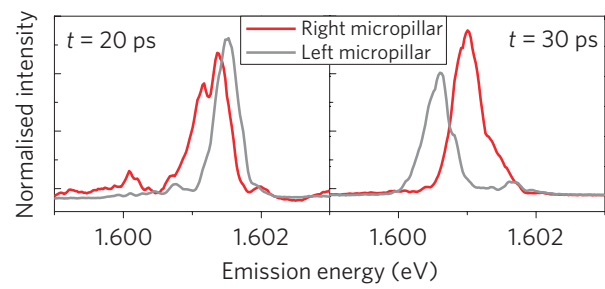

g

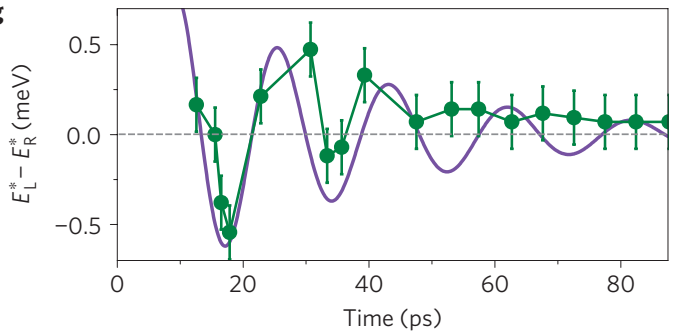

Figure 2 | Anharmonic oscillations. a, Real space emission dynamics for a high-power excitation (27 mW). b, Measured population imbalance (grey line) and phase difference (red dots), evidencing a monotonously increasing oscillation period, explicitly reported in d. The apparent damping of the oscillations observed in $\mathbf{a}, \mathbf{b}$ arises from pulse-to-pulse power fluctuations in the excitation laser. $\mathbf{c}$, Result of a simulation based on equation (2) with initial population imbalance $z(0)=0.62, \phi(0)=0, J=0.1 \mathrm{meV}, U N_{T}(t=0)=1.2 \mathrm{meV}$ and $\tau=30 \mathrm{ps}$. e, Energy- and time-resolved emission from each micropillar. The

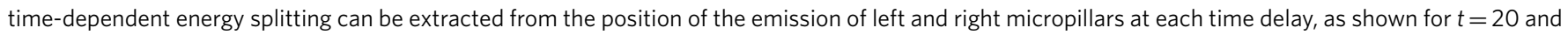

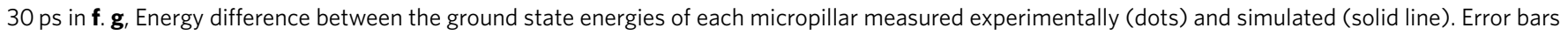
show the spectral resolution of the experiment.

across two superconductors connected by a tunnel barrier gives rise to an oscillating current ${ }^{3}$. It corresponds to the recent observation of coherent oscillations between two localized states trapped within the photonic disorder of a CdTe microcavity ${ }^{20}$.

If the initial density is large enough $\left(U N_{\mathrm{T}} \sim 2 J\right)$, interparticle repulsive interactions strongly alter the dynamics. This is the situation shown in Fig. 2, where a large polariton density induces a significant renormalization of the ground state energy of each micropillar, given by $E_{\mathrm{L}(\mathrm{R})}^{*}=E_{\mathrm{L}(\mathrm{R})}^{0}+U\left|\psi_{\mathrm{L}(\mathrm{R})}\right|^{2}$, which are blueshifted above the energy of the excitation laser (Fig. 2e). We are thus in a regime of nonlinear resonant absorption characterized by an interaction energy much larger than the tunnel coupling $J$. In these conditions we expect a dynamical self-renormalization of the energy levels in each site, when the high density of particles $\left|\psi_{\mathrm{L}(\mathrm{R})}\right|^{2}$ is transferred from one pillar to the other. We extract $E_{\mathrm{L}}^{*}, E_{\mathrm{R}}^{*}$ from the energy-resolved emission shown in Fig. 2e (see Methods), and we observe time oscillations of the energy difference $E_{\mathrm{L}}^{*}-E_{\mathrm{R}}^{*}$ (Fig. $2 \mathrm{f}-\mathrm{g}$ ). The self-induced renormalization also results in a larger bonding-antibonding splitting and, therefore, in the decrease of the oscillation period with respect to the nominal tunnelling period $2 \pi \hbar / 2 J=21 \mathrm{ps}$. This acceleration is observed at short times in Fig. 2a,b. At later times, the number of particles decreases, and so does the blueshift in each site (Fig. 2e,g). Eventually oscillations tend to recover the harmonicity with a period given by $J$ (Fig. $2 \mathrm{~d}$ ). The solution of equations (2) with the parameters of the experiment reproduces both the time evolution of $E_{\mathrm{L}}^{*}-E_{\mathrm{R}}^{*}$ and the anharmonicity of the oscillations, as shown in Fig. 2c,g.

In the high-density regime, if the initial population imbalance is prepared above the threshold ${ }^{4}, z(0)>\left(4 J / U N_{\mathrm{T}}\right)\left(\left(U N_{\mathrm{T}} / 2 J\right)-1\right)^{1 / 2}$, a strongly asymmetric renormalization of the energy takes place between the two sites (Fig. 3a). The large energy mismatch prevents the transfer of particles between the micropillars. Alternatively, we can understand this situation in the following way: the highly populated state is very close in energy and spatial distribution to the antibonding $\pi$-state. The system is thus in a quasi-metastable state in which most of the particles remain localized in one of the micropillars. This phenomenon, known as macroscopic quantum self-trapping, is reported in Fig. 3b. At short times, the large energy difference between left and right pillars results in a running phase (Fig. 3c) similar to that of Fig. 1. In the present case, differently from the a.c. Josephson effect described before, the energy difference arises entirely from self-interactions within the polariton condensates. At later times $(t>60 \mathrm{ps})$, the decay of particles reduces the energy splitting (Fig. $3 \mathrm{~d}$ ) and the oscillatoryphase regime, dominated by the tunnel coupling, is recovered (Fig. 3c). Remarkably, when oscillations set in, they take place around a phase difference value of $\pi$ (equivalently, an odd multiple 

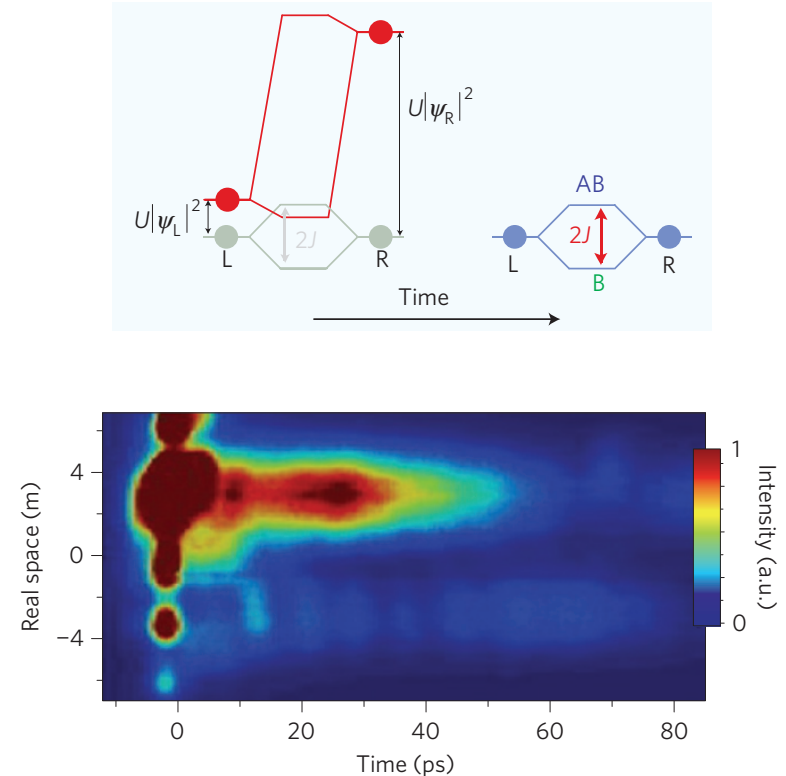

c
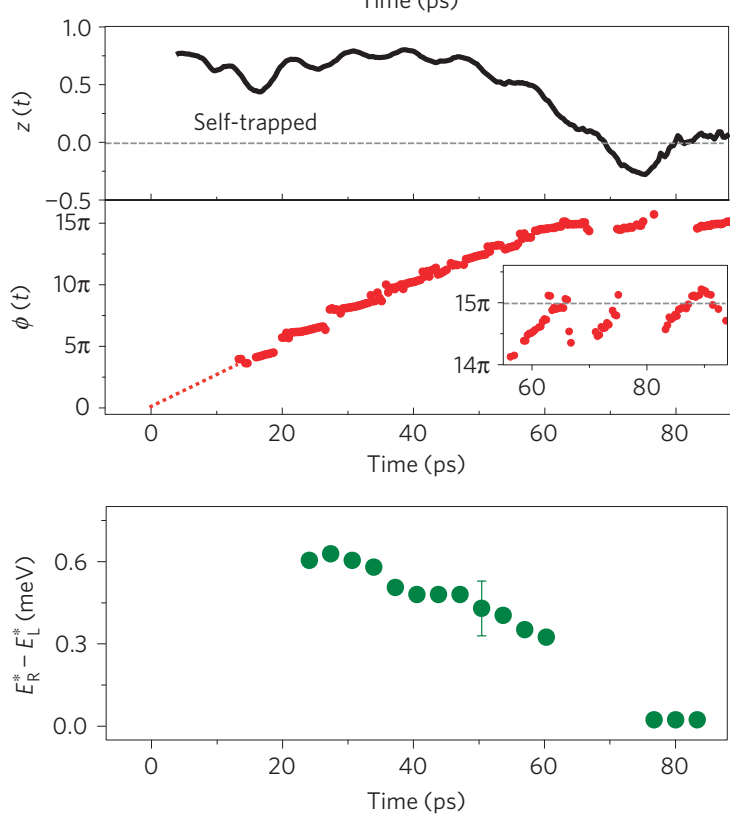

Figure $\mathbf{3}$ | Macroscopic self-trapping. a, Scheme of the renormalized energy levels at short-time (self-trapped) and long-time (harmonic oscillations) delays for a highly asymmetric excitation at high density. b. Measured dynamics of the emitted intensity. c, Time evolution of the population imbalance and phase difference. At short times particles are self-trapped in the right micropillar, and the phase difference $\phi(t)$ increases linearly with time. At $t \sim 60 \mathrm{ps}$, the escape of particles out of the microcavity induces a transition to an oscillating regime around $\phi=15 \pi$. The red dotted line is an extrapolation of the phase evolution towards $t=0$. d, Energy difference between the ground state of each micropillar extracted from the energy-resolved dynamics (see Suplementary Material). Error bars show the spectral resolution of the experiment. The energy difference is induced by the asymmetric polariton density. The transition from self-trapping to oscillations takes place when $E_{\mathrm{R}}^{*}-E_{\mathrm{L}}^{*} \sim 2 \mathrm{~J}$.

of $\pi$ ), a situation also reproduced by simulations of equations (2) (see Supplementary Information). The observed oscillations are probably damped owing to intensity fluctuations of the pump laser, resulting in fluctuations in the recovery time of the oscillations. This regime is different to the case depicted in Fig. 2, where anharmonic oscillations take place around $\phi=0$. Here, $\pi$-oscillations appear naturally from the untrapping of the condensate from the quasiantibonding mode, which is characterized by $\phi=\pi$. The dynamical transition from self-trapping to $\pi$-oscillations can be obtained only thanks to the finite particle lifetime and cannot be easily realized in systems such as Josephson junctions with atomic condensates.

In our experiments we have taken advantage of the large particleparticle interactions originating from the excitonic component of polaritons to demonstrate macroscopic self-trapping in a photonic system. This result opens the way to highly nonlinear photonic phenomena such as polarization chaos ${ }^{13}$, or spontaneous symmetry breaking and pitchfork bifurcations ${ }^{17,28,29}$, expected to give rise to highly squeezed macroscopic states ${ }^{14}$. By reducing the size of the system, the many-particle interactions evidenced here could be taken into the regime of single-photon nonlinearities ${ }^{30,31}$. Furthermore, the pair of coupled cavities we have used could be extended to arrays with minimal frequency dispersion, where photon fermionization ${ }^{32,33}$ and the emergence of Bose-Hubbard physics ${ }^{34-37}$ have been predicted.

\section{Methods}

Sample and experimental set-up. The planar sample was grown by molecular beam epitaxy and consists of a $\lambda / 2$ cavity sandwiched between two distributed Bragg reflectors containing 26 and 30 pairs, respectively, of $\mathrm{Al}_{0.95} \mathrm{Ga}_{0.01} \mathrm{As}, \mathrm{Al}_{0.20} \mathrm{Ga}_{0.80} \mathrm{As} \lambda / 4$ layers. The structure contains 3 groups of $4 \mathrm{GaAs}$ quantum wells of $70 \AA$ width, located at the maxima of the electromagnetic field in the structure, resulting in a Rabi splitting of $15 \mathrm{meV}$. The quality factor of the etched structure is 16,000 , resulting in a photon lifetime of $15 \mathrm{ps}$. As we perform the experiments in a polaritonic molecule with zero exciton-photon detuning, the polariton lifetime is extended up to $30 \mathrm{ps}$.

The polaritonic molecules are fabricated by dry etching of the planar structure. The basic building block of the molecule is a single round micropillar, which presents a set of discrete polariton levels arising from three-dimensional confinement. When two such micropillars spatially overlap, polaritons can tunnel from one micropillar to the other. The tunnel coupling is proportional to the overlap and can thus be engineered ${ }^{8}$.

Excitation of the sample is performed with a $1.7 \mathrm{ps}$ pulsed laser (repetition rate $82 \mathrm{MHz}$ ) resonant with the bonding and anti-bonding states. Its spectral width is $0.4 \mathrm{meV}$, which is larger than the bonding-antibonding splitting, thus allowing the preparation of a linear combination of both states ${ }^{26}$. Simultaneously, it is smaller than the energy separation between the ground state and the first excited state in a single micropillar ${ }^{22}$, and also smaller than the energy distance to the polariton reservoir (located $7.5 \mathrm{meV}$ above). In our analysis we can thus ignore the excited states and only consider the ground state coupled modes.

The photoluminescence dynamics are recorded using a streak camer synchronized with the excitation laser, with a time resolution of $4 \mathrm{ps}$. Recorded images are the result of integration of several million realizations. The excitation pulse is linearly polarized along the long axis of the molecule and detection is performed in the orthogonal polarization. Despite the cross-polarized detection, stray light from the laser prevents access to the population imbalance and phase difference during the first few picoseconds.

Measurement of the phase difference $\phi$. To measure the phase difference between the emissions from each micropillar, we use a Michelson interferometer. The real space emission of the molecule interferes with its mirror image at the entrance slit of a streak camera. In this way, we monitor interferences between the emission of one of the micropillars of the molecule and that of the other micropillar. Constructive/destructive interference appears depending on the phase difference between the emitted light from each micropillar, and also from the delay between the two arms of the interferometer. By varying the delay of one of the arms with a piezoelectric stage by $6 \pi$, we obtain an oscillating interference pattern from which we extract the phase difference $\phi(t)$ at different delay times ${ }^{20} t$.

Energy-resolved emission. To measure the energy of the emission, we image the micropillars on the entrance slit of a spectrometer placed in front of the streak camera. In this way we can measure the time evolution of the energy of the bonding $\left(E_{\mathrm{B}}^{*}\right)$ and antibonding $\left(E_{\mathrm{AB}}^{*}\right)$ states. From $E_{\mathrm{AB}}^{*}-E_{\mathrm{B}}^{*}$ we can calculate the energy difference between the renormalized left and right sites $E_{\mathrm{L}}^{*}, E_{\mathrm{R}}^{*}$ using the expression $E_{\mathrm{L}}^{*}-E_{\mathrm{R}}^{*}=\sqrt{\left(E_{\mathrm{AB}}^{*}-E_{\mathrm{B}}^{*}\right)^{2}-4 J^{2}}$ (see Supplementary Information).

Received 19 December 2012; accepted 15 March 2013; published online 21 April 2013

\section{References}

1. Smerzi, A., Fantoni, S., Giovanazzi, S. \& Shenoy, S. R. Quantum coherent atomic tunnelling between two trapped Bose-Einstein condensates. Phys. Rev. Lett. 79, 4950-4953 (1997). 
2. Yu, Y., Han, S., Chu, X., Chu, S-I. \& Wang, Z. Coherent temporal oscillations of macroscopic quantum states in a Josephson junction. Science 296, 889-892 (2002).

3. Josephson, B. D. Possible new effects in superconductive tunnelling. Phys. Lett. 1, 251-253 (1962).

4. Raghavan, S., Smerzi, A., Fantoni, S. \& Shenoy, S. R. Coherent oscillations between two weakly coupled Bose-Einstein condensates: Josephson effects, $\pi$ oscillations, and macroscopic quantum self-trapping. Phys. Rev. A 59, 620-633 (1999).

5. Backhaus, S. et al. Discovery of a metastable $\pi$-state in a superfluid ${ }^{3} \mathrm{He}$ weak link. Nature 392, 687-690 (1998).

6. Albiez, M. et al. Direct observation of tunnelling and nonlinear self-trapping in a single bosonic Josephson junction. Phys. Rev. Lett. 95, 010402 (2005).

7. Levy, S., Lahoud, E., Shomroni, I. \& Steinhauer, J. The a.c. and d.c. Josephson effects in a Bose-Einstein condensate. Nature 449, 579-583 (2007).

8. Galbiati, M. et al. Polariton condensation in photonic molecules. Phys. Rev. Lett. 108, 126403 (2012)

9. Sarchi, D., Carusotto, I., Wouters, M. \& Savona, V. Coherent dynamics and parametric instabilities of microcavity polaritons in double-well systems. Phys. Rev. B 77, 125324 (2008).

10. Shelykh, I. A., Solnyshkov, D. D., Pavlovic, G. \& Malpuech, G. Josephson effects in condensates of excitons and exciton polaritons. Phys. Rev. B 78, 041302 (2008).

11. Coullet, P. \& Vandenberghe, N. Chaotic self-trapping of a weakly irreversible double Bose condensate. Phys. Rev. E 64, 025202 (2001).

12. Boukobza, E., Moore, M. G., Cohen, D. \& Vardi, A. Nonlinear phase dynamics in a driven bosonic Josephson junction. Phys. Rev. Lett. 104, 240402 (2010).

13. Solnyshkov, D. D., Johne, R., Shelykh, I. A. \& Malpuech, G. Chaotic Josephson oscillations of exciton-polaritons and their applications. Phys. Rev. B 80, 235303 (2009).

14. Juliá-Díaz, B. et al. Dynamic generation of spin-squeezed states in bosonic Josephson junctions. Phys. Rev. A 86, 023615 (2012).

15. Rodrigues, A. S., Kevrekidis, P. G., Cuevas, J., Carretero-González, R. \& Frantzeskakis, D. J. Symmetry-breaking effects for polariton condensates in double-well potentials. Preprint at http://arxiv.org/abs/1205.6262 (2012).

16. Cataliotti, F. S. et al. Josephson Junction arrays with Bose-Einstein condensates. Science 293, 843-846 (2001).

17. Cambournac, C. et al. Symmetry-breaking instability of multimode vector solitons. Phys. Rev. Lett. 89, 083901 (2002).

18. Kevrekidis, P. G., Chen, Z., Malomed, B. A., Frantzeskakis, D. J. \& Weinstein, M. I. Spontaneous symmetry breaking in photonic lattices: Theory and experiment. Phys. Lett. A 340, 275-280 (2005).

19. Schmidt, S., Gerace, D., Houck, A. A., Blatter, G. \& Türeci, H. E. Nonequilibrium delocalization-localization transition of photons in circuit quantum electrodynamics. Phys. Rev. B 82, 100507(R) (2010).

20. Lagoudakis, K. G., Pietka, B., Wouters, M., André, R. \& Deveaud-Plédran, B. Coherent oscillations in an exciton-polariton Josephson junction. Phys. Rev. Lett. 105, 120403 (2010).

21. Dasbach, G., Bayer, M., Schwab, M. \& Forchel, A. Spatial photon trapping: Tailoring the optical properties of semiconductor microcavities. Semicond. Sci. Technol. 18, S339-S350 (2003).

22. Bajoni, D. et al. Polariton laser using single micropillar GaAs-GaAlAs semiconductor cavities. Phys. Rev. Lett. 100, 047401 (2008).

23. Michaelis de Vasconcellos, S. et al. Spatial, spectral, and polarization properties of coupled micropillar cavities. Appl. Phys. Lett. 99, 101103 (2011).
24. Choi, S., Morgan, S. A. \& Burnett, K. Phenomenological damping in trapped atomic Bose-Einstein condensates. Phys. Rev. A 57, 4057-4060 (1998).

25. Ciuti, C., Savona, V., Piermarocchi, C., Quattropani, A. \& Schwendimann, P. Role of the exchange of carriers in elastic exciton-exciton scattering in quantum wells. Phys. Rev. B 58, 7926-7933 (1998).

26. Paraïso, T. K. et al. Collisional damping of dipole oscillations in a trapped polariton gas. Phys. Rev. B 83, 155304 (2011).

27. Kłopotowski, Ł. et al. Optical anisotropy and pinning of the linear polarization of light in semiconductor microcavities. Solid State Commun. 139, 511-515 (2006).

28. Zibold, T., Nicklas, E., Gross, C. \& Oberthaler, M. K. Classical bifurcation at the transition from Rabi to Josephson dynamics. Phys. Rev. Lett. 105, 204101 (2010).

29. Gerving, C. S. et al. Non-equilibrium dynamics of an unstable quantum pendulum explored in a spin-1 Bose-Einstein condensate. Nature Commun. 3, 1169 (2012).

30. Verger, A., Ciuti, C. \& Carusotto, I. Polariton quantum blockade in a photonic dot. Phys. Rev. B 73, 193306 (2006).

31. Liew, T. C. H. \& Savona, V. Single photons from coupled quantum modes. Phys. Rev. Lett. 104, 183601 (2010).

32. Carusotto, I. et al. fermionized photons in an array of driven dissipative nonlinear cavities. Phys. Rev. Lett. 103, 033601 (2009).

33. Hartmann, M. J. Polariton crystallization in driven arrays of lossy nonlinear resonators. Phys. Rev. Lett. 104, 113601 (2010).

34. Angelakis, D. G., Santos, M. F. \& Bose, S. Photon-blockade-induced Mott transitions and XY spin models in coupled cavity arrays. Phys. Rev. A 76, 031805 (2007).

35. Greentree, A. D., Tahan, C., Cole, J. H. \& Hollenberg, L. C. L. Quantum phase transitions of light. Nature Phys. 2, 856-861 (2006).

36. Hartmann, M. J., Brandao, F. G. S. L. \& Plenio, M. B. Strongly interacting polaritons in coupled arrays of cavities. Nature Phys. 2, 849-855 (2006).

37. Gerace, D., Türeci, H. E., Imamoglu, A., Giovannetti, V. \& Fazio, R. The quantum-optical Josephson interferometer. Nature Phys. 5, 281-284 (2009).

\section{Acknowledgements}

We thank S. Barbay, A. Giacomotti, P. G. Kevrekidis and I. Zapata for fruitful discussions, P. Senellart for the lithography of the sample and T. Jacqmin for his experimental help. This work was supported by the French RENATECH, the ANR-11-BS10-001 contract 'QUANDYDE', the RTRA Triangle de la Physique (contract 'Boseflow1D'), the FP7 ITNs 'Clermont4' (235114) and 'Spin-Optronics' (237252), and the FP7 IRSES 'Polaphen' (246912).

\section{Author contributions}

M.A., A.A., V.G.S. and L.F. performed the experiments. M.A., A.A. and V.G.S. analysed the data. E.G., A.L. and I.S. fabricated the sample. D.D.S., H.F. and G.M. provided theoretical interpretation of the experimental results. J.B. designed and supervised the experimental work. A.A. wrote the manuscript and all authors contributed to its final version.

\section{Additional information}

Supplementary information is available in the online version of the paper. Reprints and permissions information is available online at www.nature.com/reprints. Correspondence and requests for materials should be addressed to A.A.

\section{Competing financial interests}

The authors declare no competing financial interests. 